\title{
Recovering Linear Controllability of an Underactuated Spacecraft by Exploiting Solar Radiation Pressure
}

\author{
Christopher D. Petersen* \\ University of Michigan, Ann Arbor, Michigan 48109-2140 \\ Frederick Leve‡ \\ U.S. Air Force Research Laboratory, Kirtland Air Force Base, Albuquerque, New Mexico 87117- \\ 5776 \\ and \\ Molly Flynn $¥$ and Ilya Kolmanovsky $\underline{\S}$ \\ University of Michigan, Ann Arbor, Michigan 48109-2140 \\ DOI: $\underline{10.2514 / 1 . G 001446}$
}

\begin{abstract}
This paper describes a method for recovering linear controllability for the attitude of an underactuated spacecraft by accounting for the effects of solar radiation pressure in the spacecraft attitude model. The developments are based on a spacecraft model that has at least two functional reaction wheels. A solar radiation pressure torque model that is a function of spacecraft attitude is incorporated and, under suitable assumptions, can be simplified for spacecraft with body symmetry. Conditions are given under which a symmetric-body spacecraft will experience zero solar radiation pressure torque. The stability of the underactuated spacecraft model is discussed, and necessary and sufficient conditions are given for linear controllability to be regained when solar radiation pressure torques are included in the spacecraft attitude model. With linear controllability restored, conventional controllers can be designed for underactuated spacecraft. Controllability of a cuboid spacecraft under the influence of solar radiation pressure is then analyzed. Nonlinear simulations illustrate this novel approach to recover pointing by controlling two functioning reaction wheels while two other wheels undergo subsequent failures.
\end{abstract}

$A$
$A_{w}$
$A_{j}$
$B$
$\mathcal{B}$
$C_{\mathrm{diff}, j}$
$c$
$d_{i, j}$
$d_{0}$
$\hat{g}_{i}$,
$i=1,2,3,4$
$\bar{g}_{i}$,
$i=1,2,3,4$
$\boldsymbol{H}$
$\mathcal{I}$
$I_{j}$
$J$
$\tilde{J}$
$\bar{J}$

$J_{\text {ind }}$
$J_{w}$
$J_{1}, J_{2}, J_{3}$
$L_{x}, L_{y}, L_{z}$
$l_{x}, l_{y}, l_{z}$
$\boldsymbol{M}_{\mathrm{ext}}$
$M\left(t_{f}, t_{0}\right)$
$n_{1}, n_{2}, n_{3}$
$\mathcal{O}_{\mathcal{B} / \mathcal{I}}$
$\boldsymbol{P}_{j}$
$Q, R$
$\boldsymbol{r}_{C / O}$
$\bar{r}_{C / O}$
$\bar{r}_{j}$
$\boldsymbol{r}_{j / O}$

Received 1 May 2015; revision received 2 September 2015; accepted for publication 3 September 2015; published online 24 December 2015. Copyright $\odot 2015$ by the American Institute of Aeronautics and Astronautics, Inc. All rights reserved. Copies of this paper may be made for personal and internal use, on condition that the copier pay the per-copy fee to the Copyright Clearance Center (CCC). All requests for copying and permission to reprint should be submitted to CCC at www.copyright.com; employ the ISSN 07315090 (print) or 1533-3884 (online) to initiate your request.

*Graduate Student, Department of Aerospace Engineering. Member AIAA.

${ }^{\dagger}$ Research Engineer in the Guidance, Navigation, and Control Systems Group.

${ }^{\ddagger}$ Graduate Student, Department of Aerospace Engineering; currently Research Engineer in the Guidance, Navigation, and Control Systems Group at Raytheon Missile Systems, Tucson, AZ 85756-9367.

${ }^{\S}$ Professor, Department of Aerospace Engineering. Member AIAA.
$=$ controllability index

$=$ moment of inertia of a reaction wheel about its spin axis

$=$ spacecraft principal moments of inertia

$=$ dimensions of the cuboid spacecraft

$=$ components of $\bar{r}_{C / O}$

$=$ physical external moment vector

$=$ controllability gramian

$=$ components of $\bar{u}_{s}$

$=$ orientation matrix of $\mathcal{B}$ relative to $\mathcal{I}$

$=$ physical vector of solar radiation pressure of panel $j$

$=$ weight matrices for linear quadratic controller

$=$ physical position vector of the center of mass of the spacecraft $\mathrm{C}$ from a reference point $O$

$=$ mathematical vector corresponding to $\boldsymbol{r}_{C / O}$ expressed in $\mathcal{B}$

$=$ mathematical vector corresponding to $\boldsymbol{r}_{j / O}$ expressed in $\mathcal{B}$ when considering a pair of symmetric panels

$=$ physical position vector of the center of the $j$ th panel from a reference point $O$

$=$ linearized solar radiation pressure torques influence matrix

$\bar{u}_{j} \quad=\quad$ mathematical vector corresponding to the expression of $\hat{u}_{n, j}$ expressed in $\mathcal{B}$ when considering a pair of symmetric panels

$\hat{u}_{n, j} \quad=\quad$ outward physical normal vector to panels $j$

$\hat{u}_{s} \quad=$ sun direction physical vector

$\bar{u}_{s} \quad=$ mathematical vector corresponding to the expression of $\hat{u}_{s}$ expressed in $\mathcal{I}$

$W_{a} \quad=\quad$ matrix of all operational reaction wheel spin axes

$\bar{z}_{a} \quad=\quad$ mathematical vector of control inputs corresponding to the accelerations of operational reaction wheels

$\bar{z}_{a, 0}, \quad=$ control input values at the linearization point of operational reaction wheels 


\begin{tabular}{|c|c|c|}
\hline & $=$ & $\begin{array}{l}\text { contr } \\
\text { of rea }\end{array}$ \\
\hline$\delta \bullet$ & $=$ & on from values at the linearization point \\
\hline & 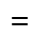 & lue of $A$ \\
\hline$\lambda_{w}$ & $=$ & de of $A_{w}$ \\
\hline $\bar{\Theta}$ & - & atical vector \\
\hline $\bar{\nu}_{a}$ & 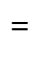 & $\begin{array}{l}\text { mathematical vector of operational reaction } \\
\text { wheels speeds }\end{array}$ \\
\hline$a, 0$ & $=$ & reaction wheel speeds at the linearization point \\
\hline$\nu_{i}, i=1,2,3,4$ & $=$ & $\begin{array}{l}\text { ) of reaction wheels } 1,2,3 \text {, } \\
y\end{array}$ \\
\hline$\tau_{p}$ & $=$ & $\begin{array}{l}\text { ar radiation pressure torque physical vector } \\
\text { uced by a pair of symmetric panels }\end{array}$ \\
\hline 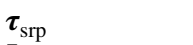 & $=$ & physical solar radiation pressure torque vector \\
\hline $\bar{\tau}_{\text {srp }}$ & $=$ & $\begin{array}{l}\text { mathematical vector corresponding to } \boldsymbol{\tau}_{\text {srp }} \\
\text { expressed in } \mathcal{B}\end{array}$ \\
\hline $\begin{array}{c}\boldsymbol{\tau}_{\mathrm{srp}, x} \\
\boldsymbol{\tau}_{\mathrm{srp}, y}, \boldsymbol{\tau}_{\mathrm{srp}, z}\end{array}$ & $=$ & $\begin{array}{l}\text { physical vectors of solar radiation pressure } \\
\text { torques aligned with the principal axes of the } \\
\text { cuboid spacecraft }\end{array}$ \\
\hline$\overline{\bar{\tau}}_{\mathrm{spp}, x}, \bar{\tau}_{\mathrm{srp}, y}, \bar{\tau}_{\mathrm{srp}, z}$ & $=$ & $\begin{array}{l}\text { mathematical vectors of } \boldsymbol{\tau}_{x, \text { srp }}, \boldsymbol{\tau}_{y, \text { srp }} \text {, and } \boldsymbol{\tau}_{z \text {,srp }} \\
\text { expressed in } \mathcal{B}\end{array}$ \\
\hline$\Phi_{\text {sun,tot }}$ & $=$ & $\mathrm{AU}$ \\
\hline$\psi, \theta$, & $=$ & 3-2-1 Euler angles yaw \\
\hline$\omega$ & $=$ & sular velocity vector \\
\hline $\bar{\omega}$ & $=$ & $\begin{array}{l}\text { mathematical vector corresponding to } \rightarrow \omega \\
\text { expressed in } \mathcal{B}\end{array}$ \\
\hline 1, & $=$ & aponents of $\bar{\omega}$ \\
\hline
\end{tabular}

\section{Introduction}

$\mathbf{T}$ HE failure of reaction wheels (RWs) in an array can impair a spacecraft's ability to perform imaging missions, during which a prescribed inertial orientation has to be achieved and maintained with high accuracy and precision. The far ultraviolet spectroscopic explorer (FUSE) had three out of its four RWs fail within their expected lifetime and consequently had to perform attitude maneuvers using magnetic actuators and one RW [1]. The Kepler telescope lost two out of its four RWs before its mission was complete. Although its gas thruster can also be used to perform attitude maneuvers, this type of actuation expends fuel and was not designed to provide the precise orientation needed [2]. The Japanese Hayabusa satellite also lost two out its four RWs. Its mission profile had to be modified to conserve fuel to have enough propellant to return to Earth after performing the necessary attitude maneuvers with gas thrusters [3]. We note that RW failures cannot be addressed simply by increasing redundancy (i.e., adding more RWs) due to stringent volume, power, and weight constraints onboard the spacecraft. Control strategies to deal with underactuated spacecraft hence need to be developed.

In the case of an underactuated spacecraft with two internal torque actuators, the linearized dynamics are not controllable. In [4], the nonlinear dynamics are shown to be inaccessible with two or fewer reaction wheels due to the conservation of angular momentum. In [5] , it is shown that if a spacecraft with two reaction wheels has zero angular momentum, the system becomes small-time locally controllable in a restricted (rest-to-rest) sense. However, the spacecraft cannot be smoothly or continuously stabilized by any time-invariant feedback law [6-9]. Stabilization is possible by time-periodic feedback laws, but exponential convergence rates cannot be achieved if the feedback law is smooth [10]. For a spacecraft with zero total angular momentum, open-loop reorientation methods are studied in [11-13], and discontinuous feedback laws are proposed in [5, 14-17].

We note that the assumption of total zero angular momentum is limiting in practical spacecraft applications. It is difficult to achieve it in space environment, and it is undesirable for spacecraft with less than four RWs because the wheels must be spun down during inertial pointing and operated in the zero crossing region, where their accuracy is decreased, the friction coefficient is increased, and their operational life is reduced.

The case of an underactuated spacecraft with nonzero total angular momentum is less studied. Boyer and Alamir [18] define a set of feasible attitudes defined by the law of angular momentum conservation and give a procedure for constructing an open-loop control. A spin-axis stabilization is performed about the uncontrollable axis of a spacecraft with nonzero angular momentum in [19], but inertial pointing is not treated. Petersen et al. [20] exploit results in [21,22] to develop a hybrid feedback control scheme, which is, however, dependent on the assumption of angular momentum conservation and does not guarantee inertial pointing in the case where there is a nonzero projection of the angular momentum on the uncontrollable spacecraft axis. The techniques proposed in the aforementioned papers not only suffer from several limitations but are also unconventional and thus unfamiliar to many practicing aerospace engineers.

The approach presented in this paper is based on including the effects of solar radiation pressure (SRP) torques, modeled following [23], into the spacecraft model. Our analysis shows that, under appropriate assumptions, linear controllability is regained, and hence spacecraft stabilization can be achieved with conventional control schemes. In particular, a linear quadratic (LQ) approach will be first applied. The LQ approach is chosen due to its robustness, its optimal control properties, and its familiarity to aerospace engineers. A pole placement scheme will also be used to improve convergence time. By taking advantage of the change in the dynamics induced by SRP torques, two RWs are able to slowly correct the attitude errors over time. This method is different from the previous approaches discussed previously. First, it exploits external disturbance torques, which many control techniques typically either neglect or reject. Second, this method is not restricted to zero total angular momentum or the constant angular momentum assumption. Third, using SRP torques allows designers to use conventional and familiar feedback control schemes that are guaranteed to locally stabilize the equilibrium.

As compared to a preliminary version of this work that has appeared in a conference paper [24], this paper contains several new results, extensions, and generalizations. These include 1) the characterization and discussion of the effects of SRP, specifically on symmetric-body spacecraft, 2) sufficient conditions for zero SRP torque acting on a symmetric spacecraft, 3) results on the fixed-attitude stability of an underactuated spacecraft with and without SRP, 4) necessary and sufficient conditions under which linear controllability is regained with SRP, and 5) extending a novel pathway to recover linear controllability and perform control of underactuated spacecraft by taking advantage of SRP torques to a broader class of spacecraft.

After [24] was submitted for publication, the press release [25] appeared, suggesting that SRP is actually used (in an unspecified control scheme) to restore Kepler's mission controllability. The controllability analysis and results in [24] and in this paper, obtained independently of [25], are thus indirectly corroborated by experimental evidence in [25].

The paper is organized as follows. The equations of spacecraft attitude kinematics and dynamics are derived in Secs. II.A and II.B. The SRP torque properties are characterized in Sec. II.C, and a linearized spacecraft model is presented in Sec. II.D. The SRP model is made specific to a symmetric spacecraft in Sec. III, and conditions under which SRP torque is zero for all attitudes for a symmetric spacecraft are subsequently derived in Sec. IV. The results on stability of an underactuated spacecraft are presented in Sec. V. Necessary and sufficient conditions for linear controllability of an underactuated spacecraft with two RWs exposed to SRP are given in Sec. VI.A, and a relative controllability metric is introduced in Sec. VI.B. The specific case of SRP torques acting on a cuboid shaped spacecraft is treated in Sec. VII and includes conditions for zero SRP torque for all attitudes as well as discussions on equilibria and controllability. The linear control design based on linear quadratic regulator theory and pole placement techniques is presented in Sec. VIII. Simulation results based on the nonlinear spacecraft model are reported in Sec. IX, where the controller is demonstrated to recover pointing after a sequence of two RW wheels failures. Concluding remarks are made in Sec. X.

Throughout this paper, the following notation is used. Frames are denoted by script, $\mathcal{S}$. A physical vector is designated by boldface italics, $\boldsymbol{r}$. Physical unit vectors are expressed with an overscript hat, $\hat{r}$. The notation for a mathematical vector obtained by resolving a physical vector in a given frame $\mathcal{S}$ is $\left.\boldsymbol{r}\right|_{\mathcal{S}}$. Mathematical vectors are 
denoted by an overscript bar, $\bar{r}$. The time derivative of a physical vector $\boldsymbol{r}$ with respect to a given frame $\mathcal{S}$ is $\boldsymbol{r}$. The orientation matrix (direction cosine matrix), representing the transformation from frame $\mathcal{S}$ to frame $\mathcal{R}$ is $\mathcal{O}_{\mathcal{R} / \mathcal{S}}$. An $m$ by $n$ zero matrix is denoted by $0_{m \times n}$, whereas an $m$ by $m$ identity matrix is denoted by $I_{m \times m}$. The nomenclature is summarized at the beginning of the paper.

\section{Spacecraft Modeling}

In this paper, a spacecraft configuration consisting of a bus and four RWs is considered. During the spacecraft's mission, two of the RWs fail and spin down to zero speed, leaving two operational RWs. Let the inertial frame be denoted by $\mathcal{I}$ and a spacecraft bus-fixed frame, with origin at the center of mass of the spacecraft bus, be denoted by $\mathcal{B}$. The axes of $\mathcal{B}$ are aligned with the spacecraft's principal axes.

All RWs rotors are assumed thin and identical, with moments of inertia about the spin axis being equal to $J_{w}$ and moments of inertia about axes transversal to the spin axis being zero. The RWs are mounted such that the center of mass of the entire spacecraft assembly is the same as that of the spacecraft bus. The four RWs spin at angular rates $\nu_{1}, \nu_{2}, \nu_{3}$, and $\nu_{4}$ about the spin axes specified by unit vectors $\hat{g}_{1}, \hat{g}_{2}, \hat{g}_{3}$, and $\hat{g}_{4}$, which are fixed in $\mathcal{B}$. Let $\bar{g}_{i}=\left.\hat{g}_{i}\right|_{\mathcal{B}}, i=1$, 2, 3, 4. Define

$$
\bar{\nu}_{a}=\left[\ldots \nu_{b} \ldots\right]^{\mathrm{T}}, \quad b \in\{1,2,3,4\}
$$

to be the vector of all operational RWs angular rates and

$$
W_{a}=\left[\ldots \bar{g}_{b} \ldots\right], \quad b \in\{1,2,3,4\}
$$

to be a matrix whose columns are the unit vectors of the spin axes of the operational RWs. For instance, if wheels 3 and 4 have failed, then $\bar{\nu}_{a}=\left[\begin{array}{ll}\nu_{1} & \nu_{2}\end{array}\right]^{\mathrm{T}}$ and $W_{a}=\left[\begin{array}{ll}\bar{g}_{1} & \bar{g}_{2}\end{array}\right]$.

\section{A. Kinematics of the Spacecraft}

The orientation of $\mathcal{B}$ relative to $\mathcal{I}$ is characterized by three successive rotations, defined by 3-2-1 Euler angles $\psi$ (yaw), $\theta$ (pitch), and $\phi$ (roll). Note that any three-parameter representation of orientation will contain singularities. It is assumed that the spacecraft's mission will not require large attitude transients and never approach the singularity. This assumption is reasonable, considering that we are interested in using RWs for accurate pointing near the target orientation. The kinematic equations, following the derivations of [26], are given by

$$
\dot{\bar{\Theta}}=F(\bar{\Theta}) \bar{\omega}
$$

where $\bar{\Theta}=\left[\begin{array}{lll}\phi & \theta & \psi\end{array}\right]^{\mathrm{T}}, \quad \bar{\omega}=\left.\boldsymbol{\omega}\right|_{\mathcal{B}}=\left[\begin{array}{lll}\omega_{1} & \omega_{2} & \omega_{3}\end{array}\right]^{\mathrm{T}} \quad$ is the angular velocity vector resolved in $\mathcal{B}$, and

$$
F(\bar{\Theta})=\frac{1}{\cos (\theta)}\left[\begin{array}{ccc}
\cos (\theta) & \sin (\phi) \sin (\theta) & \cos (\phi) \sin (\theta) \\
0 & \cos (\phi) \cos (\theta) & -\sin (\phi) \cos (\theta) \\
0 & \sin (\phi) & \cos (\phi)
\end{array}\right]
$$

\section{B. Dynamics of the Spacecraft}

The evolution of the spacecraft's angular momentum in time with respect to the inertial frame $\mathcal{I}$ is given by

$$
\stackrel{\mathcal{I}}{\boldsymbol{H}}=\stackrel{\mathcal{B}}{\boldsymbol{H}}+\boldsymbol{\omega} \times \boldsymbol{H}=\boldsymbol{M}_{\mathrm{ext}}
$$

where $\boldsymbol{H}$ is the total angular momentum of the spacecraft (i.e., including the spacecraft bus and RWs), and $\boldsymbol{M}_{\mathrm{ext}}$ is a vector of external moments about the center of mass of the spacecraft. It is assumed that the only external moments acting upon the spacecraft are torques induced by SRP (i.e., $\boldsymbol{M}_{\text {ext }}=\boldsymbol{\tau}_{\text {srp }}$ ). The total system's angular momentum expressed in $\mathcal{B}$ is

$$
\left.\boldsymbol{H}\right|_{\mathcal{B}}=J \bar{\omega}+J_{w} W_{c}\left(\bar{\nu}_{c}+W_{c}^{\mathrm{T}} \bar{\omega}\right)
$$

where

$$
\begin{aligned}
J & =\operatorname{diag}\left(J_{1}, \quad J_{2}, \quad J_{3}\right), \\
\bar{\nu}_{c} & =\left[\begin{array}{llll}
\nu_{1} & \nu_{2} & \nu_{3} & \nu_{4}
\end{array}\right]^{\mathrm{T}}, \\
W_{c} & =\left[\begin{array}{llll}
\bar{g}_{1} & \bar{g}_{2} & \bar{g}_{3} & \bar{g}_{4}
\end{array}\right]
\end{aligned}
$$

All RWs are accounted for in Eq. (6) because any residual angular rate a failed RW has before it spins down to zero affects the angular momentum of the spacecraft. Taking the time derivative of Eq. () with respect to $\mathcal{B}$ and substituting this and Eq. (6) into Eq. (5) gives

$$
J \dot{\bar{\omega}}+J_{w} W_{c}\left(\dot{\bar{\nu}}_{c}+W_{c}^{\mathrm{T}} \dot{\bar{\omega}}\right)+\bar{\omega} \times\left(J \bar{\omega}+J_{w} W_{c}\left(\bar{\nu}_{c}+W_{c}^{\mathrm{T}} \bar{\omega}\right)\right)=\bar{\tau}_{\text {srp }}
$$

where $\bar{\tau}_{\text {srp }}=\left.\tau_{\text {srp }}\right|_{\mathcal{B}}$. Let

$$
\tilde{J}=J+J_{w} W_{c} W_{c}^{\mathrm{T}}
$$

which is often referred to as the locked inertia matrix. Then, Eq. ( $\underline{8})$ simplifies to

$$
\tilde{J} \dot{\bar{\omega}}=-\bar{\omega} \times\left(\tilde{J} \bar{\omega}+J_{w} W_{c} \bar{\nu}_{c}\right)-J_{w} W_{c} \dot{\bar{\nu}}_{c}+\bar{\tau}_{\mathrm{srp}}
$$

The contribution of the failed RWs to the dynamics in Eq. (10) vanishes once their speeds reach zero. Assuming that the failed $\mathrm{RW} s$ are at zero speed, the dynamics can be rewritten to account for the contributions of only operation RWs:

$$
\bar{J} \dot{\bar{\omega}}=-\bar{\omega} \times\left(\bar{J} \bar{\omega}+J_{w} W_{a} \bar{\nu}_{a}\right)-J_{w} W_{a} \dot{\bar{\nu}}_{a}+\bar{\tau}_{\text {srp }}
$$

where

$$
\bar{J}=J+J_{w} W_{a} W_{a}^{\mathrm{T}}
$$

is the modified locked inertia matrix

The operational RW accelerations are treated as the control inputs:

$$
\dot{\bar{\nu}}_{a}=\bar{z}_{a}
$$

where

$$
\bar{z}_{a}=\left[\ldots z_{b} \ldots\right]^{\mathrm{T}}, \quad b \in\{1,2,3,4\}
$$

For instance, if wheels 1 and 2 are operational but wheels 3 and 4 have failed, $\bar{z}_{a}=\left[\begin{array}{ll}z_{1} & z_{2}\end{array}\right]^{\mathrm{T}}$.

\section{Solar Radiation Pressure Torque Model}

Assume that the spacecraft is covered with $\rho$ flat panels. The SRP torques induced by the panels are modeled based on the developments in [23]. Define

$$
\alpha_{i, j}=\frac{\Phi_{\text {sun,tot }}}{c\left(d_{i, j} / d_{0}\right)^{2}}
$$

$$
\beta_{j}=\frac{4}{9} C_{\mathrm{diff}, j}
$$

where $c$ is the speed of light, $d_{0}$ is the nominal distance from the sun equal to $1 \mathrm{AU}, \Phi_{\text {sun,tot }}$ is the solar flux at $d_{0}, C_{\text {diff, } j}$ is the diffusion coefficient for panel $j$, and $d_{i, j}$ is the distance to a point $i$ on panel $j$ from the center of the sun. The SRP at point $i$ on panel $j$ is given by

$$
\boldsymbol{P}_{i, j}=-\alpha_{i, j}\left(\hat{u}_{n, j} \cdot \hat{u}_{s}\right)\left(\hat{u}_{n, j}+\beta_{j} \hat{u}_{s}\right)
$$




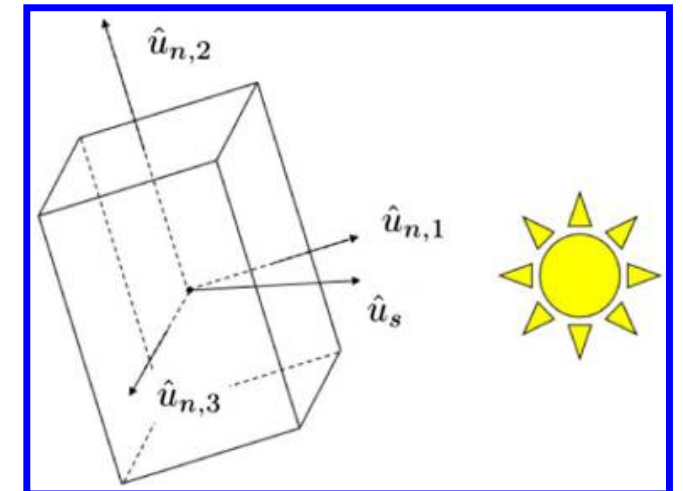

Fig. 1 Cuboid spacecraft with physical vector description.

where $\hat{u}_{n, j}$ is the normal to panel $j$ pointing outward from the spacecraft, and $\hat{u}_{s}$ is the unit vector representing the sun direction. Figure 1 depicts the sun direction $\hat{u}_{s}$ and normal vectors $\hat{u}_{n, j}, j=-1,2,3$, as they apply to a cuboid spacecraft.

The distance between two points $i$ and $l$ on panels $j$ and $k$ (where $j$ and $k$ can represent the same panel) is very small when compared to $d_{i, j}$ and $d_{l, k}$. Therefore the difference between $d_{i, j} / d_{0}$ and $d_{l, k} / d_{0}$ becomes negligible, and $\alpha_{i, j}$ can be treated as a constant parameter $\alpha$ for all points across all panels. Thus, the SRP exerted at every point along the same panel is assumed to be identical. Note that there will be a variation in SRP between panels $j$ and $k$ if $\beta_{j}$ and $\beta_{k}$ are different. The SRP exerted at each point on panel $j$ is then

$$
\boldsymbol{P}_{j}=-\alpha\left(\hat{u}_{n, j} \cdot \hat{u}_{s}\right)\left(\hat{u}_{n, j}+\beta_{j} \hat{u}_{s}\right)
$$

Under the assumption that SRP acts identically across all points on the same panel, the total SRP torque about the spacecraft's center of mass due to the $j$ th panel can be expressed as

$$
\boldsymbol{\tau}_{j, \text { srp }}=\left(\boldsymbol{r}_{j / O}-\boldsymbol{r}_{C / O}\right) \times A_{j} \boldsymbol{P}_{j}
$$

where $\boldsymbol{r}_{j / O}$ is the vector from a reference point $O$ to the center of the $j$ th panel, $\boldsymbol{r}_{C / O}$ is the vector from $O$ to the center of mass, and $A_{j}$ is the area of the $j$ th panel. Because SRP is additive across all panels,

$$
\boldsymbol{\tau}_{\mathrm{srp}}=\sum_{j}^{\rho} \boldsymbol{\tau}_{j, \mathrm{srp}} I_{j}
$$

where $I_{j}$ is an indicator function used to identify which panels are facing the sun and are acted on by solar pressure. Assuming a regular, convex shape of the spacecraft, the indicator function is given by

$$
I_{j}=\left\{\begin{array}{cc}
1 & \text { if }\left(\hat{u}_{n, j} \cdot \hat{u}_{s}\right)>0 \\
0 & \text { otherwise }
\end{array}\right.
$$

Observe that the total SRP torque in Eq. (20) is only a function of $\bar{\Theta}$. In reality, SRP is a pressure-based torque and hence, strictly speaking, also depends on spacecraft velocities due to dynamic pressure effects. These effects are very small and are assumed to be negligible.

\section{Linearized Model}

For analysis and controller design, the equations of motion [Eqs. (3) and (11)] are linearized. Without loss of generality, $\bar{\Theta}=0$ is chosen as the desired attitude because $\mathcal{I}$ can be oriented to reflect desired pointing. Theorem 1 gives the requirement for the spacecraft to maintain $\bar{\Theta}=\bar{\omega}=0$ in steady state (assuming no constraints on functioning RW speeds).

Theorem 1: $\Theta=\bar{\omega}=0$ can be maintained as a (relative) equilibrium by the closed-loop system if and only if $\bar{\tau}_{\text {srp }}(0)$ is in the range of $W_{a}$.

Proof: For the spacecraft bus, if $\bar{\omega}(t)=0$ for all $t$, then $\dot{\bar{\Theta}}(t)=0$ and $\dot{\bar{\omega}}(t)=0$. If $\dot{\bar{\omega}}=0$, then Eq. (11) implies

$$
0=-J_{w} W_{a} \bar{z}_{a}+\bar{\tau}_{\text {srp }}(0)
$$

Equation (22) can be satisfied if and only if $\bar{\tau}_{\text {srp }}(0)$ is in the range of $W_{a}$.

Remark 1: Theorem 1 gives conditions under which $\bar{\Theta}=\bar{\omega}=0$ can be maintained as a (relative) equilibrium for the spacecraft bus. The reaction wheels may be accelerating to compensate for SRP torque, in which case the (relative) equilibrium can be maintained until the RWs reach their saturation limits.

For three or more RWs, assuming that $W_{a}$ is full rank, $\bar{\Theta}=\bar{\omega}=0$ will always be a feasible equilibrium. With two or fewer RWs, Theorem 1 restricts the set of physical pointing orientations that can be maintained in steady state with functioning RWs.

Assuming that Theorem 1 holds, Eqs. (3) and (11) are linearized about $\bar{\Theta}=\bar{\omega}=0, \bar{\nu}_{a}=\bar{\nu}_{a, 0}$, and $\bar{z}_{a}=\overline{\bar{z}}_{a, 2}=\bar{\Xi}$, where $\Xi$ is the commanded RW accelerations to make $\Theta=\bar{\omega}=0$ a feasible equilibrium, i.e.,

$$
\Xi= \begin{cases}\left(W_{a}^{\mathrm{T}} W_{a}\right)^{-1} W_{a}^{\mathrm{T}} \bar{\tau}_{\text {srp }}(0) & \text { if } \operatorname{rank}\left(W_{a}\right) \leq 3, \\ \text { an arbitrary } \bar{z}_{a} \text { such that } W_{a} \bar{z}_{a}=\bar{\tau}_{\text {srp }}(0) & \text { otherwise }\end{cases}
$$

Assuming that there are $m$ operational RWs and the failed RWs are at zero speed, the linearization yields the system of equations

$$
\left[\begin{array}{c}
\dot{\bar{\Theta}} \\
\dot{\bar{\omega}}
\end{array}\right]=\left[\begin{array}{cc}
0_{3 \times 3} & I_{3 \times 3} \\
\bar{J}^{-1} T & \bar{J}^{-1} J_{w} S\left[W_{a} \bar{\nu}_{a, 0}\right]
\end{array}\right]\left[\begin{array}{c}
\bar{\Theta} \\
\bar{\omega}
\end{array}\right]+\left[\begin{array}{c}
0_{3 \times m} \\
-\bar{J}^{-1} J_{w} W_{a}
\end{array}\right] \delta \bar{z}_{a}
$$

where $\delta \bar{z}_{a}=\bar{z}_{a}-\bar{z}_{a, 0}, T$ is the linearized matrix of $\bar{\tau}_{\text {srp }}$ (i.e., $\left.\bar{\tau}_{\text {srp }}=\bar{\tau}_{\text {srp }}(0)+T \bar{\Theta}\right)$, and $S[*]$ is the $3 \times 3$ skew-symmetric matrix formed off a $3 \times 1$ mathematical vector. That is, if $\bar{a}=\left[\begin{array}{lll}a_{1} & a_{2} & a_{3}\end{array}\right]^{\mathrm{T}}$, then

$$
S[\bar{a}]=\left[\begin{array}{ccc}
0 & -a_{3} & a_{2} \\
a_{3} & 0 & -a_{1} \\
-a_{2} & a_{1} & 0
\end{array}\right]
$$

Letting $\bar{x}=\left[\begin{array}{ll}\bar{\Theta} & \bar{\omega}\end{array}\right]^{\mathrm{T}}$, it follows that

$$
\dot{\bar{x}}=A \bar{x}+B \delta \bar{z}_{a}
$$

where

$$
A=\left[\begin{array}{ccc}
0_{3 \times 3} & I_{3 \times 3} & \\
\bar{J}^{-1} T & \bar{J}^{-1} J_{w} S\left[W_{a}\right. & \left.\bar{\nu}_{a, 0}\right]
\end{array}\right], \quad B=\left[\begin{array}{c}
0_{3 \times m} \\
-\bar{J}^{-1} J_{w} W_{a}
\end{array}\right]
$$

\section{Solar Radiation Pressure Torque on a Symmetric- Body Spacecraft}

To provide insight into the effects of SRP, a general class of symmetric-body spacecraft that have pairs of panels with the same area and diffusion coefficients (i.e., same $\beta_{j}$ ), located on the opposite ends of the spacecraft, is now considered. The panels are equal distance away from the reference point $O$, and the normals to the panels are parallel but opposite in direction. Denote one panel in this pair by " $p+$ " and another by " $p-$ ". Then,

$$
\begin{gathered}
\boldsymbol{r}_{p+/ O}=-\boldsymbol{r}_{p-/ O}=\boldsymbol{r}_{p}, \\
\hat{u}_{n, p+}=-\hat{u}_{n, p-}=\hat{u}_{p}, \\
\beta_{p+}=\beta_{p-}=\beta_{p}, \\
A_{p-}=A_{p+}=A_{p}
\end{gathered}
$$

The SRP torque exerted by each individual panel, assuming they are exposed to the sun, is 


$$
\begin{aligned}
\boldsymbol{\tau}_{p+} & =\left(\boldsymbol{r}_{p+}-\boldsymbol{r}_{C / O}\right) \times A_{p+} \boldsymbol{P}_{p+}, \\
& =\gamma_{p}\left(\left(\boldsymbol{r}_{p} \times \hat{u}_{p}\right)+\beta_{p}\left(\boldsymbol{r}_{p} \times \hat{u}_{s}\right)-\left(\boldsymbol{r}_{C / O} \times \hat{u}_{p}\right)-\beta_{p}\left(\boldsymbol{r}_{C / O} \times \hat{u}_{s}\right)\right)
\end{aligned}
$$

$$
\begin{aligned}
\boldsymbol{\tau}_{p-} & =\left(\boldsymbol{r}_{p-}-\boldsymbol{r}_{C / O}\right) \times A_{p-} \boldsymbol{P}_{p-}, \\
& =\gamma_{p}\left(-\left(\boldsymbol{r}_{p} \times \hat{u}_{p}\right)+\beta_{p}\left(\boldsymbol{r}_{p} \times \hat{u}_{s}\right)-\left(\boldsymbol{r}_{C / O} \times \hat{u}_{p}\right)+\beta_{p}\left(\boldsymbol{r}_{C / O} \times \hat{u}_{s}\right)\right)
\end{aligned}
$$

where $\gamma_{p}=-\alpha A_{p}\left(\hat{u}_{p} \cdot \hat{u}_{s}\right)$. The total SRP torque induced by the two panels is

$$
\tau_{p}=\tau_{p+} I_{p+}+\tau_{p-} I_{p-}
$$

By the assumption on directions of $\hat{u}_{p+}$ and $\hat{u}_{p-}, I_{p+}$ takes on the opposite binary value of $I_{p-}$ if $\gamma_{p} \neq 0$. If $\gamma_{p}=0$, then the pair of panelsAU: The sentence that begins "If $\gamma \mathrm{p}=0$, then ..." is unclear, specifically the portion that reads "the pair of panels does to induce." Please review this sentence carefully, and edit/revise as necessary. does to induce any SRP torque. Knowing this, and noting that the second and third terms of Eqs. (29) and (30) are the same, whereas the first and fourth terms differ by a sign, Eq. (31) can be written as

$$
\begin{aligned}
\boldsymbol{\tau}_{p} & =\gamma_{p}\left(\left(-\boldsymbol{r}_{C / O} \times \hat{u}_{p}\right)+\beta_{p}\left(\boldsymbol{r}_{p} \times \hat{u}_{s}\right)+\operatorname{sign}\left(\hat{u}_{p} \cdot \hat{u}_{s}\right)\left(\left(\boldsymbol{r}_{p} \times \hat{u}_{p}\right)\right.\right. \\
& \left.\left.-\beta_{p}\left(\boldsymbol{r}_{C / O} \times \hat{u}_{s}\right)\right)\right)
\end{aligned}
$$

\section{Conditions for Zero Solar Radiation Pressure Torque for All Orientations}

There are cases in which the total SRP torque exerted on the spacecraft is zero for all possible orientations. Hence, utilization of SRP for control is not possible. Theorem 2 gives a condition for which a symmetric-body spacecraft covered in pairs of symmetric panels will experience zero SRP torque, regardless of $\bar{\Theta}$.

Theorem 2: Let a symmetric-body spacecraft be covered with $\rho_{s}$ pairs of panels, having characteristics satisfying Eq. (28). Then, the SRP torque induced on the spacecraft is zero for all $\Theta$ if the three relations

$$
\begin{gathered}
\boldsymbol{r}_{i} \| \hat{u}_{i}, i=1 \ldots \rho_{s}, \\
\boldsymbol{r}_{C / O}=0, \\
\exists a \in \mathbb{R}: \sum_{i=1}^{\rho_{s}} \beta_{i} A_{i} \bar{r}_{i} \bar{u}_{i}^{\mathrm{T}}=a I_{3 \times 3}
\end{gathered}
$$

are all satisfied, where $\bar{r}_{i}=\left.\boldsymbol{r}_{i}\right|_{\mathcal{B}}$ and $\bar{u}_{i}=\left.\hat{u}_{i}\right|_{\mathcal{B}}$.

Proof: The total SRP torque exerted on the symmetric spacecraft can be written using Eq. (32):

$$
\begin{aligned}
\boldsymbol{\tau}_{\text {srp }} & =\sum_{i=1}^{\rho_{s}} \gamma_{i}\left(\left(-\boldsymbol{r}_{C / O} \times \hat{u}_{i}\right)+\beta_{i}\left(\boldsymbol{r}_{i} \times \hat{u}_{i}\right)\right. \\
& \left.+\operatorname{sign}\left(\hat{u}_{i} \cdot \hat{u}_{s}\right)\left(\left(\boldsymbol{r}_{i} \times \hat{u}_{i}\right)-\beta_{i}\left(\boldsymbol{r}_{C / O} \times \hat{u}_{s}\right)\right)\right)
\end{aligned}
$$

If the first two conditions of Eq. (33) hold, Eq. (34) becomes

$$
\boldsymbol{\tau}_{\mathrm{srp}}=\sum_{i=1}^{\rho_{s}} \gamma_{i} \beta_{i}\left(\boldsymbol{r}_{i} \times \hat{u}_{i}\right)
$$

Let $\bar{u}_{s}=\left.\hat{u}_{s}\right|_{\mathcal{I}}$. Resolving Eq. (35) in $\mathcal{B}$ yields

$$
\bar{\tau}_{\text {srp }}=-\alpha\left(\left(\sum_{i=1}^{\rho_{s}} \beta_{i} A_{i} \bar{r}_{i} \bar{u}_{i}^{\mathrm{T}}\right) \mathcal{O}_{\mathcal{B} / \mathcal{I}} \bar{u}_{s}\right) \times\left(\mathcal{O}_{\mathcal{B} / \mathcal{I}} \bar{u}_{s}\right)
$$

If the third condition of Eq. (33) holds, then there exists an $a \in \mathbb{R}$ such that

$$
\bar{\tau}_{\text {srp }}=-a \alpha \mathcal{O}_{\mathcal{B} / \mathcal{I}} \bar{u}_{s} \times \mathcal{O}_{\mathcal{B} / \mathcal{I}} \bar{u}_{s}=0
$$

Therefore, regardless of orientation, the SRP will not exert any torques on the spacecraft. $\square$

An example of when Theorem 2 holds is for a cuboid spacecraft where the reference point $O$ coincides both with the center of mass and the center of solar radiation pressure of the spacecraft. See Sec. VII.A

\section{Stability of a Spacecraft}

In general, the open-loop spacecraft attitude dynamics for a fixedattitude equilibrium without SRP are unstable, though the dynamics of the angular velocities can be stable depending on the axis of rotation [27]. As we discuss in this section, a fixed-attitude equilibrium of an underactuated spacecraft with SRP has similar open-loop instability properties.

\section{A. Linear Stability with Zero Solar Radiation Pressure}

Consider the case of an underactuated spacecraft with two operational RWs, where effects of SRP torques are zero (i.e., $\boldsymbol{\tau}_{\text {srp }}=0$ ). The state matrix $A$ in Eq. (27) becomes

$$
A=\left[\begin{array}{ccc}
0_{3 \times 3} & I_{3 \times 3} & \\
0_{3 \times 3} & \bar{J}^{-1} J_{w} S\left[W_{a}\right. & \left.\bar{\nu}_{a, 0}\right]
\end{array}\right]
$$

The eigenvalues of matrix $A$, denoted as $\lambda$, are given by

$$
\lambda=0,0,0,0, \pm J_{w} \sqrt{\left(W_{a} \bar{\nu}_{a, 0}\right)^{\mathrm{T}} D\left(W_{a} \bar{\nu}_{a, 0}\right)}
$$

where $D=-\left(\operatorname{diag}\left(J_{2} J_{3}, \quad J_{1} J_{3}, \quad J_{1} J_{2}\right)\right)^{-1}$. The matrix $D$ is negative definite, and therefore, $A$ might have one pair of purely imaginary eigenvalues, provided that at least one of the RW speeds at the equilibrium is nonzero. Therefore, all eigenvalues lie on the imaginary axis.

The requirement for stability of a linear system with eigenvalues on the imaginary axis and in the open left half-plane is that each such eigenvalue with zero real part must be semisimple (i.e., the geometric multiplicity of the eigenvalue must be equal to its algebraic multiplicity). There is only one pair of purely imaginary eigenvalues, and so both are semisimple. Any instability may thus be caused only by the zero eigenvalue. It can be shown that the algebraic multiplicity of the zero eigenvalue of $A$ is 4 , whereas its geometric multiplicity is 3 . Thus, the linearized system is open-loop unstable.

We note that the angular velocity variables are decoupled from the orientation variables for this linearized system. The state matrix for the angular velocities, denoted as $A_{\omega}$, is given by

$$
A_{w}=\bar{J}^{-1} J_{w} S\left[W_{a} \bar{\nu}_{a, 0}\right]
$$

whose eigenvalues $\lambda_{w}$ are

$$
\lambda_{w}=0, \pm J_{w} \sqrt{\left(W_{a} \bar{\nu}_{a, 0}\right)^{\mathrm{T}} D\left(W_{a} \bar{\nu}_{a, 0}\right)}
$$

All the eigenvalues of $A_{w}$ are semisimple, and hence it can be shown that the linearized angular velocity dynamics are stable but not asymptotically stable. Because of the double integrator structure of $A$, bounded angular velocities may cause unbounded drift in the attitude, resulting in instability.

\section{B. Stability Analysis of Linearized Underactuated Spacecraft Dynam-} ics with Solar Radiation Pressure Torque

We now consider the case with nonzero SRP torque (i.e., $\boldsymbol{\tau}_{\text {srp }} \neq 0$ ) and two functioning RWs spinning about the first and second principal axes. We analyze the stability properties of the spacecraft 
bus (relative) equilibrium corresponding to $\bar{\Theta}=\bar{\omega}=0$. It is assumed that $\bar{\tau}_{\text {srp }}(0)$ is consistent with Theorem 1 so that the (relative) equilibrium can be maintained. Let the RW accelerations be set according to the following control law:

$$
\delta \bar{z}_{a}=-\left(C^{\mathrm{T}} C\right)^{-1} C^{\mathrm{T}} \bar{J}^{-1} T \bar{\Theta}
$$

where $C=-\left(\bar{J}^{-1} J_{w} W_{a}\right)$. The feedback law in Eq. (42) cancels out the SRP torque components that are in the range of $\bar{W}_{a}$. Then, the dynamics matrix has the following form:

$$
A=\left[\begin{array}{cc}
0_{3 \times 3} & I_{3 \times 3} \\
\tilde{T} & \bar{J}^{-1} J_{w} S\left[W_{a} \bar{\nu}_{a, 0}\right]
\end{array}\right]
$$

where

$$
\tilde{T}=\left[\begin{array}{ccc}
0 & 0 & 0 \\
0 & 0 & 0 \\
\tilde{t}_{31} & \tilde{t}_{32} & \tilde{t}_{33}
\end{array}\right]
$$

and $\tilde{t}_{3 j} \in \mathbb{R}, j=1,2,3$. Computing the eigenvalues of $A_{c}$ yields three zero eigenvalues, a pair of complex eigenvalues, and an eigenvalue that is purely real (the equations for these eigenvalues are omitted due to lack of space). The zero eigenvalue has a geometric multiplicity of 2 and is, therefore, not semisimple. Hence, the equilibrium is unstable based on the linearized model. In addition, as apparent from Eq. (26), the SRP torque causes a coupling between the attitude and angular velocity dynamics, which does not allow the independent analysis of the stability of angular velocity dynamics.

\section{Nonlinear Stability}

The previous analysis is based on the linear model in Eq. (26). Because the linearized model has eigenvalues on the imaginary axis, the stability analysis is inconclusive because the stability/instability of an equilibrium in such a case may depend on nonlinear terms. Note that, without SRP, there do exist stable equilibria where the spacecraft can maintain directional pointing while rotating about an axis parallel to the pointing direction [27]. However, the previous analysis suggests that a fixed-attitude equilibrium either with or without SRP can be unstable. Intuitively, if one of the angular velocities is perturbed away from zero, a bounded drift in the Euler angles away from equilibrium can occur. This instability conclusion, supported by the linear analysis, has been verified through extensive simulations on a nonlinear model. The formal mathematical proof of instability (e.g., based on an application of Chetaev's theorem [28]) is left to future work.

\section{Regaining Linear Controllability Using Solar Radiation Pressure}

\section{A. Necessary and Sufficient Conditions for Regaining Linear}

\section{Controllability}

Without the effects of SRP included in Eq. (26), the spacecraft dynamics are linearly uncontrollable by RW accelerations if only two RWs are functioning. The following theorem gives necessary and sufficient conditions for restoring linear controllability to a spacecraft with two functioning RWs when SRP torques are included.

Theorem 3: Let the spacecraft have two operational RWs whose spin axis are nonparallel, i.e., $\operatorname{rank}\left(W_{a}\right)=2$. The system [Eq. (26)] is linearly controllable if and only if, for every eigenvalue $\lambda$ of $A$ and for any vector $\bar{\eta}_{u}$ in the null space of $W_{a}^{\mathrm{T}}$,

$$
\bar{\eta}_{u}^{\mathrm{T}}\left(\bar{J} \lambda^{2}-S\left[W_{a} \bar{\nu}_{a, 0}\right] \lambda-T\right) \neq 0
$$

Proof: The Popov-Belevitch-Hautus test for controllability implies that Eq. (26) is linearly controllable if and only if

$$
\operatorname{rank}\left[\left(\lambda I_{6 \times 6}-A\right) \quad B\right]=\operatorname{dim}(A)
$$

for every eigenvalue $\lambda$ of A; see [29]. By the converse, the system in Eq. (26) is uncontrollable if and only if there exists a nonzero vector $\bar{\eta} \in \overline{\mathbb{C}^{6}}$ such that

$$
\bar{\eta}^{*}\left[\begin{array}{ll}
\left(\lambda I_{6 \times 6}-A\right) & B
\end{array}\right]=0
$$

for at least one eigenvalue, where $\bar{\eta}^{*}$ is the conjugate transpose of $\bar{\eta}$. Equation (47) can be reduced to two conditions:

$$
\bar{\eta}^{*}\left(\lambda I_{6 \times 6}-A\right)=0, \quad \bar{\eta}^{*} B=0
$$

Let $\bar{\eta}=\left[\begin{array}{ll}\bar{\eta}_{1}^{*} & \bar{\eta}_{2}^{*}\end{array}\right]^{*}, \quad \bar{\eta}_{1}, \bar{\eta}_{2} \in \mathbb{C}^{3}$. Expanding the first condition of Eq. (요), it follows that

$$
\bar{\eta}_{1}^{*} \lambda-\bar{\eta}_{2}^{*} \bar{J}^{-1} T=0, \quad-\bar{\eta}_{1}^{*}+\bar{\eta}_{2}^{*}\left(I_{3 \times 3}-\bar{J}^{-1} J_{w} S\left[W_{a} \bar{\nu}_{a, 0}\right]\right)=0
$$

Multiplying the second part of Eq. (49) by $\lambda$ and adding it together with the first part of Eq. (49) gives

$$
\bar{\eta}_{2}^{*}\left(I_{3 \times 3} \lambda^{2}-\bar{J}^{-1} J_{w} S\left[W_{a} \bar{\nu}_{a, 0}\right]-\bar{J}^{-1} T\right)=0
$$

The second condition of Eq. (48) can be simplified to

$$
\bar{\eta}_{2}^{*} \bar{J}^{-1} J_{w} W_{a}=0
$$

Taking the conjugate transpose of Eq. (51), and noting that $W_{a}^{*}=W_{a}^{\mathrm{T}}, J_{w}^{*}=J_{w}$, and $\left(\bar{J}^{-1}\right)^{*}=\bar{J}^{-1}$, yields

$$
J_{w} W_{a}^{\mathrm{T}} \bar{J}^{-1} \bar{\eta}_{2}=0
$$

Let $\tilde{\eta}_{2}$ be the real part of $\bar{\eta}_{2}$. Then, $\bar{J}^{-1} \tilde{\eta}_{2}$ is in the null space of $W_{a}^{\mathrm{T}}$. Denoting $\bar{\eta}_{u}=\bar{J}^{-1} \tilde{\eta}_{2}$, Eq. (무) can be written as

$$
\bar{\eta}_{u}^{\mathrm{T}}\left(\bar{J} \lambda^{2}-S\left[W_{a} \bar{\nu}_{a, 0}\right] \lambda-T\right)=0
$$

Thus, for Eq. (26) to be uncontrollable, there must exist an $\bar{\eta}_{u}$ in the null space of $W_{a}^{\mathrm{T}}$ and an eigenvalue $\lambda$ such that Eq. (53) holds. Reversing the arguments, it can be similarly shown that if, for each eigenvalue of $A$, Eq. (53) cannot be satisfied with $\bar{\eta}_{u}$ in the null space of $W_{a}^{\mathrm{T}}$, then the system is controllable.

Corollary 3.1: Theorem 3 can be simplified for the case when there are two operational RWs about the first two principal moments of inertia, i.e.,

$$
W_{a}=\left[\begin{array}{ll}
1 & 0 \\
0 & 1 \\
0 & 0
\end{array}\right]
$$

Let $t_{3, i}, i=1,2,3$ be the $i$ th entry of the third row of $T$. Because $\tilde{\eta}_{u}$ is in the null of $W_{a}^{\mathrm{T}}, \bar{\eta}_{u}=\left[\begin{array}{lll}0 & 0 & \eta\end{array}\right]^{\mathrm{T}}$, where $\eta \in \mathbb{R}$. Equation (53) can be rewritten as

$$
\eta \lambda^{2}\left[\begin{array}{lll}
0 & 0 & J_{3}
\end{array}\right]-\eta \lambda\left[-J_{w} \nu_{0,2} \quad J_{w} \nu_{0,1} \quad 0\right]=\eta\left[\begin{array}{lll}
t_{31} & t_{32} & t_{33}
\end{array}\right]
$$

which gives the following three equations:

$$
J_{3} \lambda^{2}=t_{33}, \quad J_{w} \nu_{0,2} \lambda=t_{31}, \quad J_{w} \nu_{0,1} \lambda=-t_{32}
$$

For the system to be uncontrollable, the three parts of Eq. (56) must all hold for at least one eigenvalue of $A$. By the converse, if for each eigenvalue of $A$, one of the parts in Eq. (53) is not satisfied (it does not have to be the same equation for each eigenvalue), the system is controllable.

Theorem 4 is a consequence of Theorem 3 and provides a sufficient condition for an underactuated system to remain linearly uncontrollable even if SRP torques are added into the math model.

Theorem 4: Let the spacecraft be equipped with two operational RWs whose spin axes are not parallel. The system [Eq. (26)] is linearly uncontrollable if $\bar{\eta}_{u}^{\mathrm{T}} T=0$ for $\bar{\eta}_{u}$ in the null space of $\bar{W}_{a}^{\mathrm{T}}$. 
Proof: Suppose that, for $\bar{\eta}_{u}$ in the null space of $W_{a}^{\mathrm{T}}$,

$$
\bar{\eta}_{u}^{\mathrm{T}} T=0
$$

By Theorem 3, if there exists an eigenvalue $\lambda$ of $A$ such that

$$
\bar{\eta}_{u}^{\mathrm{T}}\left(\bar{J} \lambda^{2}-S\left[W_{a} \bar{\nu}_{a, 0}\right] \lambda-T\right)=0
$$

then the system is uncontrollable. Equation (57) simplifies Eq. (58) to

$$
\bar{\eta}_{u}^{\mathrm{T}}\left(\bar{J} \lambda^{2}-S\left[W_{a} \bar{\nu}_{a, 0}\right] \lambda\right)=0
$$

If Eq. (57) holds, then $T$ is not full rank, which implies that $A$ is not full rank and contains a zero eigenvalue. The eigenvalue $\lambda=0$ satisfies Eq. (59), and therefore the system is uncontrollable. $\square$

Remark 2: The results in Theorem 3, Corollary 3.1, Theorem 4, and numerical examples suggest that the dynamics of the spacecraft with two RWs and SRP effects included are linearly controllable in a broad range of cases. Theorem 4 indicates that the linearized SRP torque must have nonzero projection on the uncontrolled direction for linear controllability to hold.

\section{B. Relative Controllability}

To assess the relative controllability of different spacecraft configurations, we consider the following controllability index:

$$
J_{\text {ind }}=\lambda_{\max }\left(e^{A^{\mathrm{T}}\left(t_{f}-t_{0}\right)} M\left(t_{f}, t_{0}\right)^{-1} e^{A\left(t_{f}-t_{0}\right)}\right)
$$

where $\lambda_{\max }$ denotes the maximum eigenvalue of a matrix, $t_{0}$ is the initial time of the maneuver, $t_{f}$ is the final time of the maneuver, and $M\left(t_{f}, t_{0}\right)$ denotes the controllability gramian. The controllability index $J_{\text {ind }}$ corresponds to the maximum effort (where effort is defined as the minimum of the integral of the input squared) required to bring an initial state $x\left(t_{0}\right)$ of unit norm to zero. Note that the metric is defined over a finite time interval, given that the system is open-loop unstable. This metric will be used later to assess the controllability of the cuboid spacecraft.

\section{Solar Radiation Pressure Effects on a Cuboid Spacecraft}

Suppose that the spacecraft of interest is a cuboid, which is a commonly used spacecraft shape. This spacecraft has a symmetric body and has dimensions $L_{x}, L_{y}$, and $L_{z}$. Let $\hat{u}_{1}, \hat{u}_{2}$, and $\hat{u}_{3}$ be the normals to the panels covering the spacecraft sides, which, because the structure of the cuboid, are parallel to the principal axes. The reference point $O$ is chosen as the geometric center of the cuboid spacecraft. Using the notation from Sec. III, the parameters to obtain the SRP torque are defined as

$$
\begin{aligned}
& \bar{r}_{1}=\left[\begin{array}{c}
\frac{L_{x}}{2} \\
0 \\
0
\end{array}\right], \quad \bar{r}_{2}=\left[\begin{array}{c}
0 \\
\frac{L_{y}}{2} \\
0
\end{array}\right], \quad \bar{r}_{3}=\left[\begin{array}{c}
0 \\
0 \\
\frac{L_{z}}{2}
\end{array}\right] \\
& \bar{u}_{1}=\left[\begin{array}{l}
1 \\
0 \\
0
\end{array}\right], \quad \bar{u}_{2}=\left[\begin{array}{l}
0 \\
1 \\
0
\end{array}\right], \quad \bar{u}_{3}=\left[\begin{array}{l}
0 \\
0 \\
1
\end{array}\right] \\
& A_{1}=L_{y} L_{z}, \quad A_{2}=L_{x} L_{z}, \quad A_{3}=L_{x} L_{y}
\end{aligned}
$$

The center of mass of the spacecraft will also be offset from $O$, and

$$
\bar{r}_{C / O}=\left.\boldsymbol{r}_{C / O}\right|_{\mathcal{B}}=\left[\begin{array}{lll}
l_{x} & l_{y} & l_{z}
\end{array}\right]^{\mathrm{T}} \text {. }
$$

This offset is of particular importance because it influences the relative controllability of the system.

\section{A. Conditions for Zero Solar Radiation Pressure}

Based on Theorem 2, it may be shown that, under certain conditions, the cuboid spacecraft can experience zero SRP torque for all $\bar{\Theta}$. By the physical structure of the cuboid spacecraft, $\boldsymbol{r}_{i} \| \hat{u}_{i}$, $i=1,2,3$, and so the first condition of Eq. (33) in Theorem 2 is satisfied. If $O$ is aligned with the center of mass (i.e., $\boldsymbol{r}_{C / O}=0$ ), the second condition of Eq. (33) is satisfied. Assume that the diffusion coefficient is the same for all panels $\left(\beta_{1}=\beta_{2}=\beta_{3}=\beta\right)$. Then, using the definitions in Eqs. (61-63),

$$
\sum_{i=1}^{3} \beta_{i} A_{i} \bar{r}_{i} \bar{u}_{i}^{\mathrm{T}}=\frac{\beta L_{x} L_{y} L_{z}}{2} I_{3 \times 3}
$$

which satisfies the third condition of Eq. (33) with $a=L_{x} L_{y} L_{z} / 2$. Therefore, by Theorem 2, if $\boldsymbol{r}_{C / O}=0$ and all panels have the same diffusion properties, a cuboid spacecraft will experience zero SRP torque. It should be noted that, if $\beta_{i}, i=1,2,3$, were different, the conditions for zero SRP torque given by Theorem 2 may not hold, and it may be possible to take advantage of SRP torques to recover linear controllability.

\section{B. Equilibrium Analysis}

Let $\boldsymbol{\tau}_{\mathrm{srp}, x}, \boldsymbol{\tau}_{\mathrm{srp}, y}$, and $\boldsymbol{\tau}_{\mathrm{srp}, z}$ be the SRP torques exerted on the cuboid spacecraft by panels whose normals are parallel with the principal axes. Suppose that $\bar{u}_{s}=\left[\begin{array}{lll}n_{1} & n_{2} & n_{3}\end{array}\right]^{\mathrm{T}}$, and let $\bar{\Theta}=0$. Then the $\mathrm{SRP}$ torques exerted on the spacecraft, given by Eq. (32), resolved in $\mathcal{B}$, are

$$
\begin{aligned}
& \left.\boldsymbol{\tau}_{\mathrm{srp}, x}\right|_{\mathcal{B}}=\bar{\tau}_{\mathrm{srp}, x} \\
& =\frac{A_{1} \alpha n_{1}}{2}\left[\begin{array}{c}
0 \\
2 l_{z}+L_{x} \beta n_{3} \\
-2 l_{y}-L_{x} \beta n_{2}
\end{array}\right]+\operatorname{sign}\left(n_{1}\right) A_{1} \alpha n_{1} \beta\left[\begin{array}{c}
l_{y} n_{3}-l_{z} n_{2} \\
l_{z} n_{1}-l_{x} n_{3} \\
l_{x} n_{2}-l_{y} n_{1}
\end{array}\right]
\end{aligned}
$$

$$
\begin{aligned}
\left.\boldsymbol{\tau}_{\text {srp }, y}\right|_{\mathcal{B}}= & \bar{\tau}_{\text {srp }, y} \\
& =\frac{A_{2} \alpha n_{2}}{2}\left[\begin{array}{c}
-2 l_{z}-L_{y} \beta n_{3} \\
0 \\
2 l_{x}+L_{y} \beta n_{1}
\end{array}\right]+\operatorname{sign}\left(n_{2}\right) A_{2} \alpha n_{2} \beta\left[\begin{array}{l}
l_{y} n_{3}-l_{z} n_{2} \\
l_{z} n_{1}-l_{x} n_{3} \\
l_{x} n_{2}-l_{y} n_{1}
\end{array}\right]
\end{aligned}
$$

$$
\begin{aligned}
& \left.\boldsymbol{\tau}_{\mathrm{srp}, z}\right|_{\mathcal{B}}=\bar{\tau}_{\mathrm{srp}, z} \\
& \quad=\frac{A_{3} \alpha n_{3}}{2}\left[\begin{array}{c}
2 l_{y}+L_{z} \beta n_{2} \\
-2 l_{x}-L_{z} \beta n_{1} \\
0
\end{array}\right]+\operatorname{sign}\left(n_{3}\right) A_{3} \alpha n_{3} \beta\left[\begin{array}{l}
l_{y} n_{3}-l_{z} n_{2} \\
l_{z} n_{1}-l_{x} n_{3} \\
l_{x} n_{2}-l_{y} n_{1}
\end{array}\right]
\end{aligned}
$$

The conditions for $\bar{\Theta}=\bar{\omega}=0$ to be a feasible closed-loop (relative) equilibrium are given by Theorem 1 and supported by Eqs. (66-68). Suppose, for instance, $l_{x}=l_{z}=0$ while $l_{y}$ is nonzero, whereas the operational RWs have spin axes aligned with the first and second principal axes. If $n_{1}=0, n_{2}=1$, and $n_{3}=0$, then based on the aforementioned expressions, $\tau_{\text {srp }}(0)=0$, and the spacecraft can remain at the relative equilibrium with $\bar{\Theta}=\bar{\omega}=0$ without any RWs accelerating. If the possibility of RWs accelerating to maintain the (relative) equilibrium at $\bar{\Theta}=\bar{\omega}=0$ is acceptable (e.g., to enable the spacecraft to obtain images while compensating for nonzero SRP with RWs), then the condition can be relaxed. In this case, as long as $n_{1}=0$ (i.e., the third principal axis is not pointed toward the sun), the SRP torque is of the form $\tau_{\text {srp }}(0)=\left[\begin{array}{lll}*, & *, & 0\end{array}\right]^{\mathrm{T}}$ and can be 
Table 1 Model parameters

\begin{tabular}{|c|c|c|}
\hline$\underline{\text { Parameter }}$ & Units & Value \\
\hline$J$ & $\mathrm{~kg} \cdot \mathrm{m}^{2}$ & $\operatorname{diag}(430,1210,1300)$ \\
\hline$J_{w}$ & $\mathrm{~kg} \cdot \mathrm{m}^{2}$ & 0.043 \\
\hline$L_{x}$ & $\mathrm{~m}$ & 2 \\
\hline$L_{y}$ & $\mathrm{~m}$ & 2.5 \\
\hline$L_{z}$ & $\mathrm{~m}$ & 5 \\
\hline$l_{x}$ & $\mathrm{~m}$ & 0 \\
\hline$l_{y}$ & $\mathrm{~m}$ & 0.1 and 0.5 \\
\hline$l_{z}$ & $\mathrm{~m}$ & 0 \\
\hline \multirow[t]{3}{*}{$W$} & - - & {$\left[\begin{array}{llll}1 & 0 & 0 & 1 / \sqrt{3}\end{array}\right.$} \\
\hline & & $\begin{array}{llll}0 & 1 & 0 & 1 / \sqrt{3}\end{array}$ \\
\hline & & {$\left[\begin{array}{llll}0 & 0 & 1 & 1 / \sqrt{3}\end{array}\right]$} \\
\hline$\Phi_{\text {sun }}$ & $\mathrm{W} / \mathrm{m}^{2}$ & 1367 \\
\hline$C_{\text {diff }, j}$ & -- & 0.2 \\
\hline$c$ & $\mathrm{~m} / \mathrm{s}$ & 299792458.0 \\
\hline
\end{tabular}

compensated by the available RWs that are along the first and second principal axes.

\section{Relative Controllability of the Cuboid Spacecraft}

To demonstrate controllability of the cuboid spacecraft, it is assumed that the spacecraft has parameters listed in Table 1, the first and second RWs are operational, and $\bar{\nu}_{a, 0}=\left[\begin{array}{ll}100 & 100\end{array}\right]^{\mathrm{T}} \mathrm{rad} / \mathrm{s}$. The third and fourth RWs are assumed to have previously failed and are now at zero speed. The plot of $J_{\text {ind }}$ in Eq. (60) versus $l_{y}$ and the time of the maneuver $t_{f}\left(t_{0}=0\right)$ is given in Fig. 2. The spacecraft is more controllable for larger $l_{y}$, and the control effort decreases if longer maneuver time is available. Intuitively, more SRP torque is produced when the distance between the center of pressure and the center of mass is greater. This torque can be exploited to enhance controllability.

\section{Controller Design}

Because the linearized dynamics of a spacecraft body with certain asymmetry properties are linearly controllable with the addition of SRP torques to the spacecraft model, a controller based on conventional linear quadratic (LQ) theory [30] can be used to stabilize the system to equilibrium. The LQ controller minimizes the cost

$$
J_{L Q}=\int_{0}^{\infty} x(\tau)^{\mathrm{T}} Q x(\tau)+\delta \bar{z}_{a}(\tau)^{\mathrm{T}} R \delta \bar{z}_{a}(\tau) \mathrm{d} \tau
$$

where $Q=Q^{\mathrm{T}} \geq 0$ and $R=R^{\mathrm{T}}>0$ are weighting matrices. The controller has the following form:

$$
\delta \bar{z}_{a}=K_{L Q} x
$$

where $K_{L Q}$ is the LQ gain.

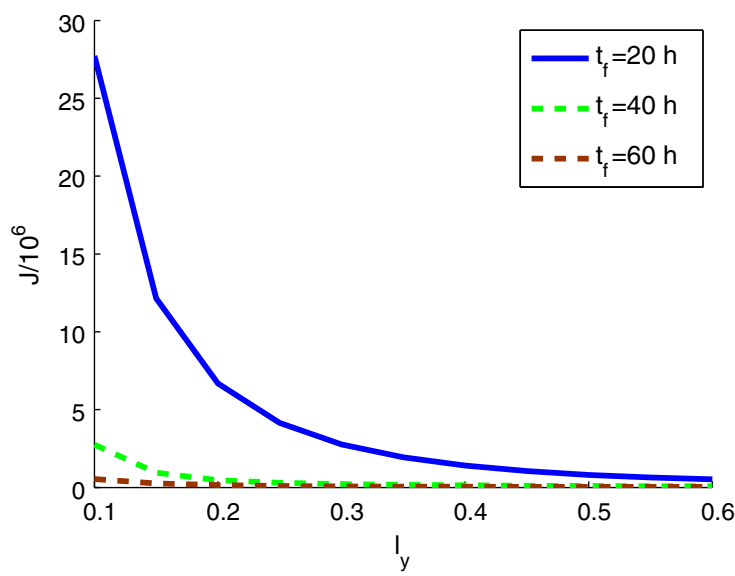

Fig. 2 Controllability metric (scaled by $10^{6}$ ) vs $l_{y}$ and maneuver time, $t_{f}$.
In the subsequent simulations, $R=1000 I_{m \times m}$, where $m$ is the number of operational RWs. If $m \geq 3, Q=$ $\operatorname{diag}(10,10,10,0.01,0.01,0.01)$ is used. If $m=2$ and the only operational RWs spin about the first and second principal axes, $Q=\operatorname{diag}(40,10,10,0.04,0.01,0.01)$ is used to emphasize faster regulation of $\phi$ and $\omega_{1}$, which improves the transient response.

\section{Results}

In this section, nonlinear simulations are presented where four wheels are initially operational. Two separate wheel failures occur during these simulations: one wheel fails at $5 \mathrm{~h}$ and the other at $20 \mathrm{~h}$. The speed response of a failed wheel spinning down is modeled by a first-order lag with a 10 min settling time. The simulations are run on the full nonlinear model of the spacecraft kinematics and dynamics, including the nonlinear model of SRP torques. All spacecraft parameters are given by Table 1 . Nominally, $\bar{u}_{s}=\left[\begin{array}{lll}0 & 1 & 0\end{array}\right]^{\mathrm{T}}$, and simulation results are presented for two different sequences of wheel failures. Additional simulation results are then reported for the case when $\bar{u}_{s}=[01 / \sqrt{2} 1 \sqrt{2}]^{\mathrm{T}}$, creating a situation where the RWs must accelerate to maintain spacecraft pointing. Finally, convergence times from different initial conditions are quantified.

\section{A. Wheel 3 Fails First}

The first case considered is when RW 3 fails first, followed by RW 4. The offset of the center of pressure from the center of mass is given by $l_{y}=0.5 \mathrm{~m}$ and $l_{x}=l_{z}=0 \mathrm{~m}$. The responses are shown in Fig. 3 . There is a much larger disturbance to orientation when the second wheel fails. The controller manages the first wheel failure quickly, then reconfigures and handles the second wheel failure over a longer period of time, coordinating two operational RWs in presence of SRP.

\section{B. Wheel 4 Fails First, Reduced $l_{y}$}

Now, the case is considered when $l_{y}$ is reduced to $0.1 \mathrm{~m}$ so that the spacecraft is less controllable (see Sec. VI.B). In this simulation, RW 4 fails first, followed by RW 3. The responses are shown in Fig. 4. The controller is able to manage the failures and reduce the spacecraft orientation error over time. However, the responses are slower due to worse spacecraft controllability.

\section{Wheel 4 Fails First, Skewed Pointing}

In this case study, which is referred to as the skewed pointing, $l_{y}=0.1 \mathrm{~m}$ while $\bar{u}_{s}=[01 / \sqrt{2} 1 \sqrt{2}]^{\mathrm{T}}$. The controller is modified with an additional feedforward term, which cancels out $\bar{\tau}_{s r p}(0)$. Note that the third component of $\bar{\tau}_{\mathrm{srp}}(0)$ is zero; hence, canceling the steady-state value of SRP torque by the acceleration of RWs 1 and 2 is feasible. The response is shown in Fig. 5. Observe that the controller handles RW 3 and 4 failures and that RWs 1 and 2 continue to accelerate to be able to maintain the spacecraft orientation in steady state with desired pointing.

\section{Achieving Faster Closed-Loop Response Time}

A faster linear controller may be designed using the pole placement method. As confirmed by simulations, more aggressive controllers, however, have smaller regions of attraction (ROAs) for the nonlinear system and are not able to always recover after the simulated sequence of wheel failures. If spacecraft thrusters (which are not as precise as reaction wheels) are employed to first reduce the attitude and angular velocity errors before the two functional RWs are used, the implementation of more aggressive controllers for RWs becomes feasible. Toward this end, a pole-placement-based linear controller was designed with the closed-loop poles of $-0.0137 \pm$ $0.0068 i,-0.0208 \pm .0021 i,-0.0001$, and -0.0075 (versus $-0.0012 \pm 0.0068 i, \quad-0.0019 \pm .0021 i, \quad-6.4906 \times 10^{-6}, \quad$ and -0.007 for the nominal LQ controller), and Monte-Carlo simulations were performed to characterize the closed-loop response from various initial conditions. For each simulation, the initial Euler angles were selected from a uniform distribution in the interval [-2, 2 deg], and the angular velocity was initially zero. Only two RWs (those aligned with the first two principal axes) were assumed to be 


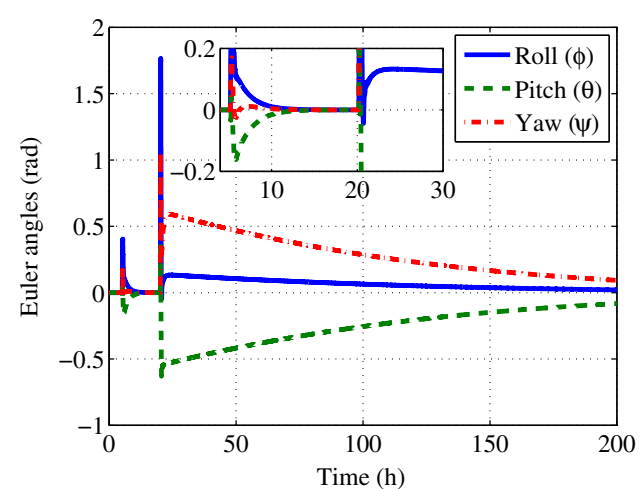

a)

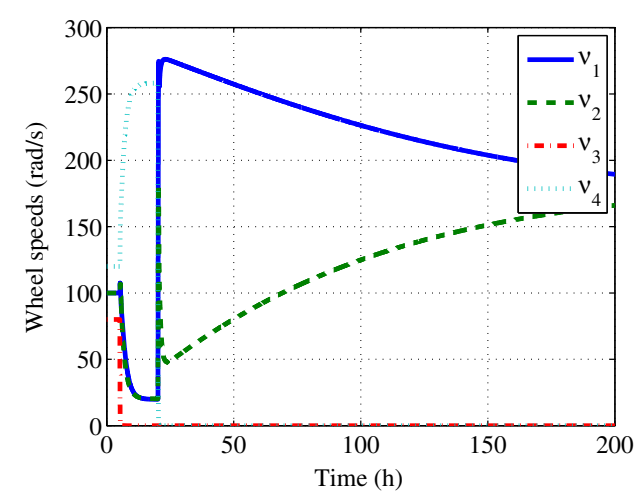

c)

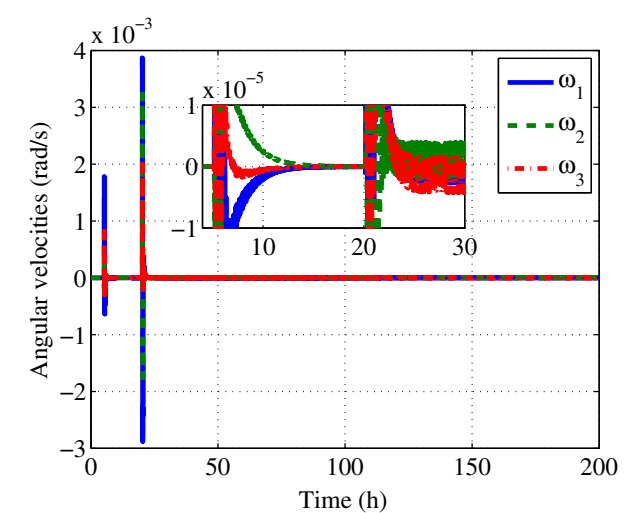

b)

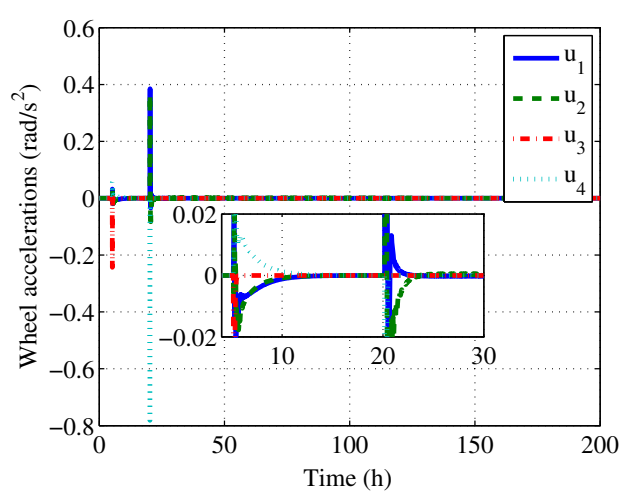

d)

Fig. 3 Representations of a) Euler angles, b) angular velocities, c) RW speeds, and d) $R W$ accelerations in the nonlinear simulation when wheel 3 fails first, $l_{y}=0.5 \mathrm{~m}$.

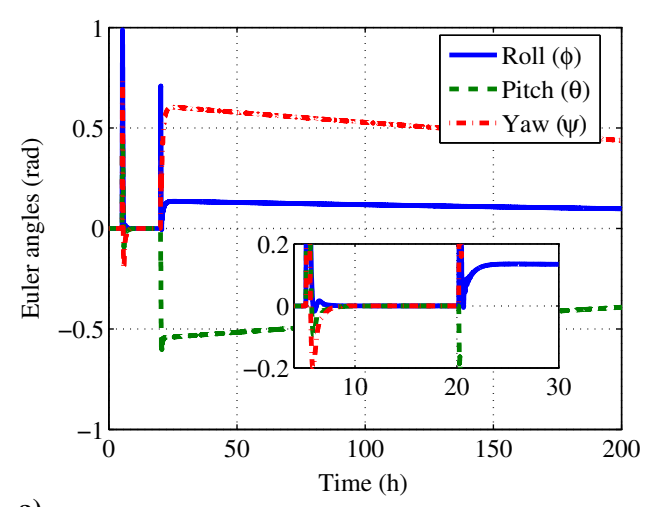

a)

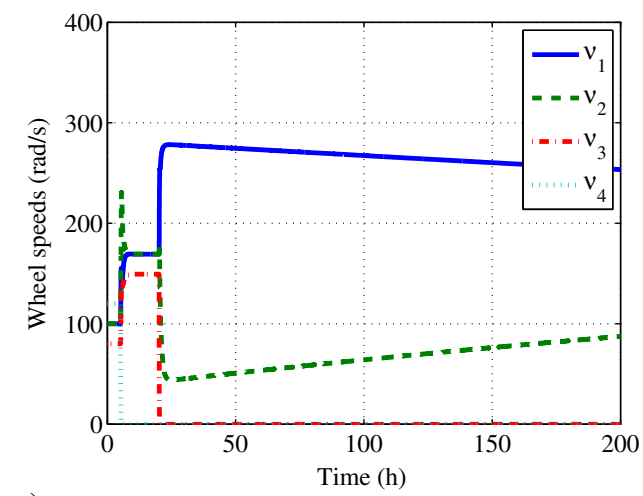

c)

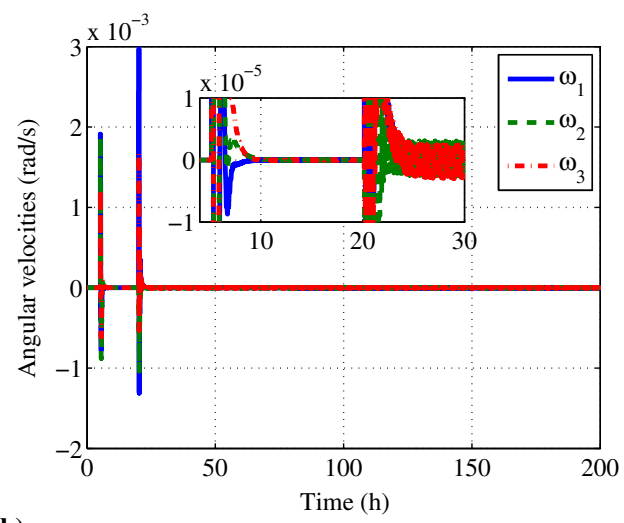

b)

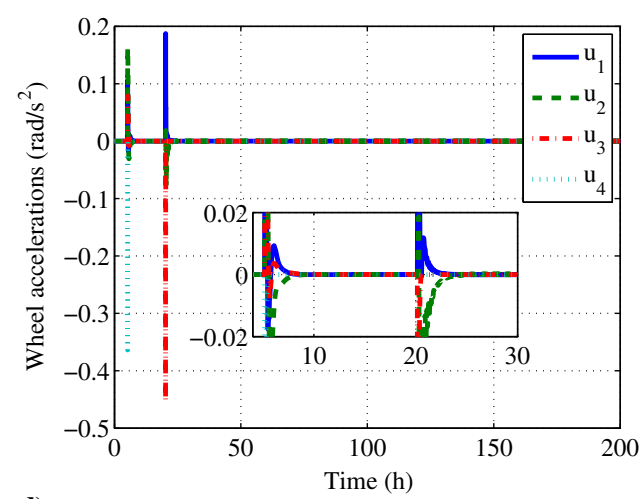

d)

Fig. 4 Representations of a) Euler angles, b) angular velocities, c) RW speeds, and d) $R W$ accelerations in the nonlinear simulation when wheel 4 fails first, $l_{y}=0.1 \mathrm{~m}$. 


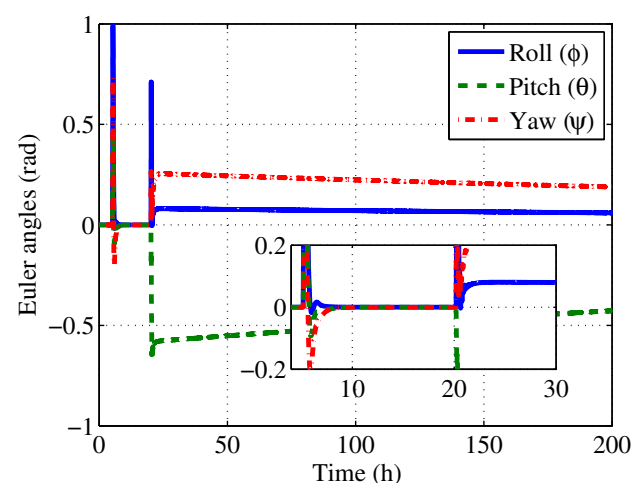

a)

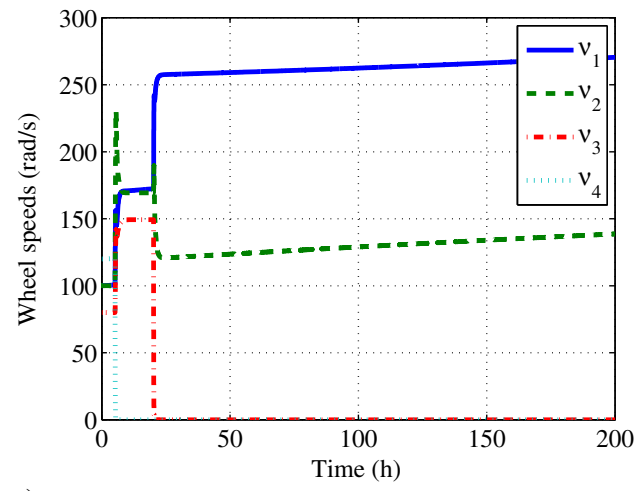

c)

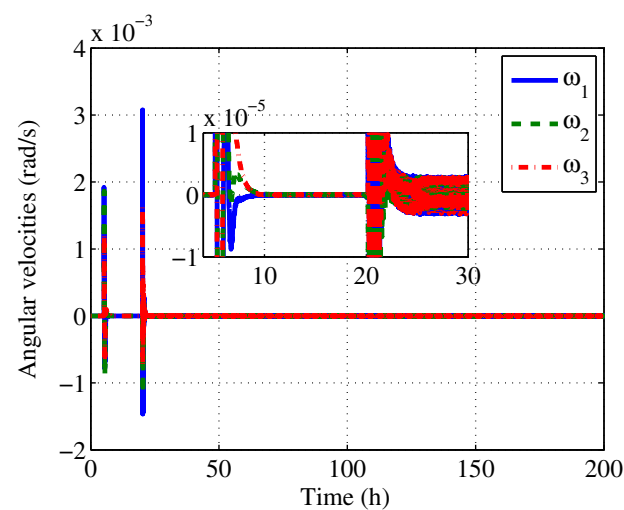

b)

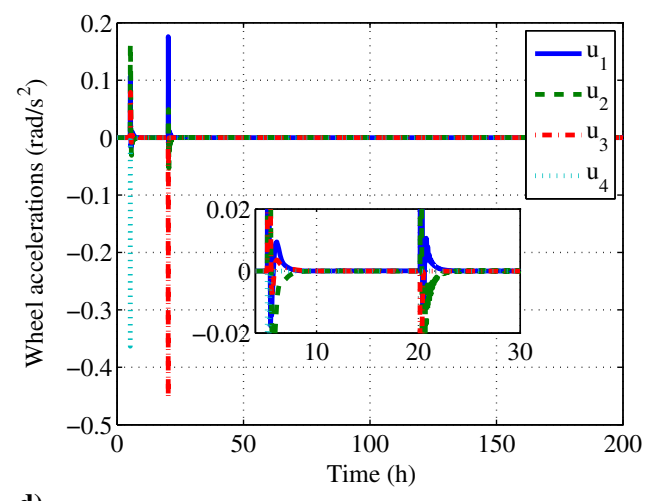

d)

Fig. 5 Representations of a) Euler angles, b) angular velocities, c) RW speeds, and d) RW accelerations in the nonlinear simulation when wheel 4 fails first, skewed pointing.

functional, each initially rotating at a speed of $100 \mathrm{rad} / \mathrm{s} .1900$ simulation runs were performed. The average time it took for the spacecraft orientation to enter a $0.001 \mathrm{deg}$ box around the equilibrium was $35.1554 \mathrm{~h}$, with a standard deviation of $4.2532 \mathrm{~h}$. In all cases, the controller was convergent and able to bring the Euler angles to the target box. The maximum angular speed and accelerations of the wheels in these simulations were $247.5929 \mathrm{rad} / \mathrm{s}$ and $9.7833 \mathrm{rad} / \mathrm{s}^{2}$, respectively, which are within actuator capability limits.

\section{E. Regions of Attractions}

Each controller has associated with it a unique ROA such that if the spacecraft starts within this region, the controller will stabilize the system to equilibrium. Such regions can be estimated by using scaled sublevel sets of the Lyapunov function for the linearized system, but these estimates may be quite conservative [31]. The ROA in our case cannot be easily or analytically described for an arbitrary spacecraft because the dynamics depend on the shape of spacecraft, the desired

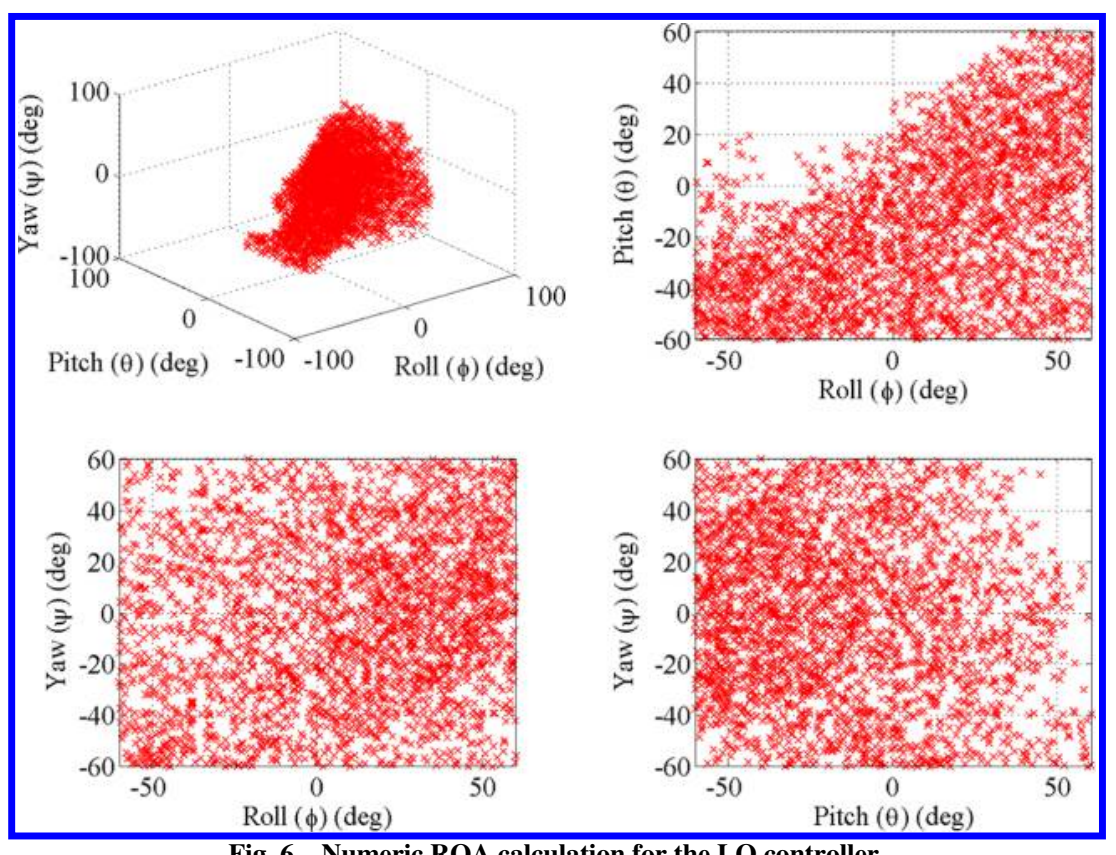

Fig. 6 Numeric ROA calculation for the LQ controller. 

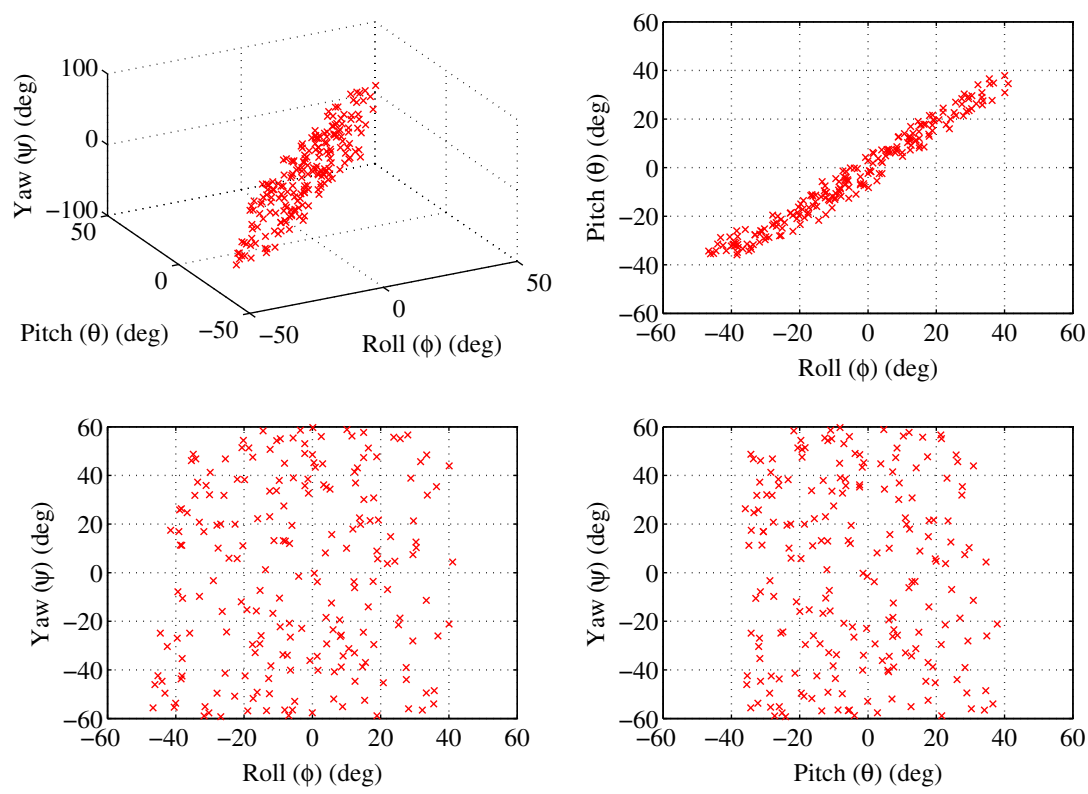

Fig. 7 Numeric ROA calculation for the pole placement controller.

inertial pointing direction, the spin axis directions of operational RWs, and the chosen control scheme. Therefore, nonlinear simulations are used to approximate the ROA for a cuboid spacecraft using the LQ and the pole placement controllers in this work. 5000 random test simulations were run using each controller with initial Euler angles belonging to the interval of [ $-60,60 \mathrm{deg}]$, initial zero angular velocity, and RW speeds initially at $100 \mathrm{rad} / \mathrm{s}$. The operational RWs are aligned with the first and second principal axes. The results for both controllers are shown in Figs. 6 and 7, in which the X's designate all the initial attitudes that the controllers can stabilize to the desired pointing equilibrium. As is to be expected, the LQ controller has a larger ROA than the more aggressive pole placement controller.

\section{Conclusions}

This paper treated an inertial pointing attitude control problem for an underactuated spacecraft with two reaction wheels. An unconventional pathway to recover linear controllability was demonstrated through incorporating solar radiation pressure torques into the spacecraft model. For certain fixed inertial pointing directions and realistic spacecraft configurations, linear controllability is recovered, and spacecraft control becomes feasible with conventional linear quadratic and pole placement techniques. Although the maneuvers take time, the approach presented is not restricted to the assumption of zero angular momentum; in fact, because of the presence of solar radiation pressure torques, the total angular momentum may not be zero and is not conserved. The results open up the possibility of applying a variety of conventional control techniques to the underactuated spacecraft control problem.

\section{Acknowledgments}

The authors gratefully acknowledge the partial contribution from the U.S. Air Force Office of Scientific Research under the lab task LRIR11RV15COR for this research.

\section{References}

[1] Moos, H. W., "Overview of the Far Ultraviolet Spectroscopic Explorer Mission," Astrophysical Journal Letters, Vol. 38, No. 12, July 2000, pp. L1-L6.

[2] Cowen, R., "The Wheels Come off Kepler," Nature, Vol. 497, No. 7450, May 2013, pp. 417-418.

doi: $10.1038 / 497417 \mathrm{a}$.
[3] de Selding, P. B., "Japan's Asteroid Sample-Return Mission Has Problems," Space.com, Oct. 2005, http://www.space.com/1642-japanasteroid-sample-return-mission-problems.html [retrieved July 2015].

[4] Crouch, P., "Spacecraft Attitude Control and Stabilization: Applications of Geometric Control Theory to Rigid Body Models," Transactions on Automatic Control, Vol. 29, No. 4, 1984, pp. 321-332. doi:10.1109/TAC.1984.1103519

- [5] Krishnan, H., McClamroch, H., and Reyhanoglu, M., "Attitude Stabilization of a Rigid Spacecraft Using Two Momentum Wheel Actuators," Journal of Guidance, Control, and Dynamics, Vol. 18, No. 2, 1995, pp. 256-263. doi: $10.2514 / 3.21378$

[6] Brockett, R. W., "Asymptotic Stability and Feedback Stabilization," Differential Geometric Control Theory, edited by Brockett, R. W., Millman, R. S., and Sussmann, H. J., Birkhauser, Boston, 1983, pp. 181-191.

[7] Byrnes, C. I., and Isidori, A., "On the Attitude Stabilization of Rigid Spacecraft," Automatica, Vol. 27, No. 12, 1991, pp. 87-95. doi:10.1016/0005-1098(91)90008-P

[8] Krishnan, H., Reyhanoglu, M., and McClamroch, H., "Attitude Stabilization of a Rigid Spacecraft Using Two Control Torques: A Nonlinear Control Approach Based on the Spacecraft Attitude Dynamics," Automatica, Vol. 30, No. 6, 1994, pp. 1023-1027. doi:10.1016/0005-1098(94)90196-1

[9] Zabczyk, J., "Some Comments on Stabilizability," Applied Mathematics and Optimization, Springer-Verlag, New York, 1989, pp. 1-9.

[10] Gurvits, L., and Li, Z., "Smooth Time-Periodic Feedback Solutions for Nonholonomic Motion Planning," Progress in Nonholonomic Motion Planning, Springer, New York, 1993, pp. 53-108.

[11] Rui, C., Kolmanovsky, I., and McClamroch, N. H., "Nonlinear Attitude and Shape Control of Spacecraft with Articulated Appendages and Reaction Wheels," Transactions on Automatic Control, Vol. 45, No. 8 , Aug. 2000, pp. 1455-1469. doi: $10.1109 / 9.871754$

[12] Zidek, R., and Kolmanovsky, I., "Approximate Optimal Control of Nonlinear Systems with Quadratic Performance Criteria," Proceedings of the American Control Conference, IEEE Publ., Piscataway, NJ, 2015, pp. 5587-5592. doi:10.1109/ACC.2015.7172214

[13] Ge, X., and Chen, L., "Optimal Reorientation of Underactuated Spacecraft Using Genetic Algorithm with Wavelet Approximation," World Congress on Intelligent Control and Automation, Vol. 6, June 2004, pp. 5466-5470. doi:10.1109/WCICA.2004.1343777

[14] Gui, H., Jin, L., and Xu, S., "Attitude Maneuver Control of a TwoWheeled Spacecraft With Bounded Wheel Speeds," Acta Astronautica, Vol. 88, July-Aug. 2013, pp. 98-107. doi:10.1016/j.actaastro.2013.03.006

[15] Horri, N. M., and Hodgart, S., "Attitude Stabilization of an Underactuated Satellite Using Two Wheels," Proceedings of the IEEE 
Aerospace Conference, Vol. 6, IEEE Publ., Piscataway, NJ, Nov. 2003, pp. 2629-2635.

doi:10.1109/AERO.2003.1235188

[16] Horri, N. M., and Palmer, P., "Practical Implementation of Attitude-Control Algorithms for an Underactuated Satellite," Journal of Guidance, Control, and Dynamics, Vol. 35, No. 12, 2012, pp. $40-50$.

doi:10.2514/1.54075

[17] Urakubo, T., Tsuchiya, K., and Tsujita, K., "Attitude Control of a Spacecraft with Two Reaction Wheels," Journal of Vibration and Control, Vol. 10, No. 9, 2004, pp. 1291-1311. doi:10.1177/1077546304042042

[18] Boyer, F., and Alamir, M., "Further Results on the Controllability of a Two-Wheeled Satellite," Journal of Guidance, Control, and Dynamics, Vol. 30, No. 2, March-April 2007, pp. 611-619. doi:10.2514/1.21505

[19] Kim, S., and Kim, Y., "Spin-Axis Stabilization of a Rigid Spacecraft Using Two Reaction Wheels," Journal of Guidance, Control, and Dynamics, Vol. 24, No. 5, 2001, pp. 1046-1049. doi: $10.2514 / 2.4818$

[20] Petersen, C., Leve, F., and Kolmanovsky, I., "Hybrid Switching Attitude Control of Underactuated Spacecraft Subject to Solar Radiation Pressure," Proceedings of the Spaceflight Mechanics Meeting, American Astronautical Soc. Paper 15-327, Springfield, VA, 2015.

[21] Kolmanovsky, I., and McClamroch, N. H., "Hybrid Feedback Laws for a Class of Cascade Nonlinear Control Systems," Transactions on Automatic Control, Vol. 41, No. 9, 1996, pp. 1217-1282. doi:10.1109/9.536497

[22] Rui, C., Kolmanovsky, I., and McClamroch, H., "Hybrid Control for Stabilization of a Class of Cascade Nonlinear Systems," Proceedings of the American Control Conference, Vol. 5, IEEE Publ., Piscataway,
NJ, June 1997, pp. 2800-2804.

doi:10.1109/ACC.1997.611966

[23] Richard, L., Jah, M. K., Crassidis, J. L., Leve, F. A., and Kelecy, T., "Astrometric and Photometric Data Fusion for Inactive Space Object Mass and Area Estimation," Acta Astronautica, Vol. 99, JuneJuly 2014, pp. 1-15. doi:10.1016/j.actaastro.2013.10.018

[24] Flynn, M., Leve, F., Petersen, C., and Kolmanovsky, I., "Linear Control of Underactuated Spacecraft with Two Reaction Wheels Made Feasible by Solar Radiation Pressure," Proceedings of the American Control Conference, IEEE Publ., Piscataway, NJ, 2015, pp. 3193-3198. doi:10.1109/ACC.2015.7171824

[25] Howell, E., "Kepler K2 Finds First Exoplanet, a Super-Earth, While Surfing Sun's Pressure Wave for Control," Universe Today, Courtenay, British Columbia, Dec. 2014, http://www.universetoday.com/117462 [retrieved Feb. 2015].

[26] Hughes, P., "Rotational Kinematics," " Spacecraft Attitude Dynamics, edited by Hughes, P., Dover, Mineda, NY, 2004, pp. 18-28.

[27] Hughes, P., "Attitude Dynamics of a Rigid Body," Spacecraft Attitude Dynamics, edited by Hughes, P., Dover Publ., Mineda, NY, 2004 pp. 121-124.

[28] Khalil, H., "Lyapunov Stability," Nonlinear Systems, 3rd ed., PrenticeHall, Upper Saddle River, NJ, 2002, pp. 111-181.

[29] Chen, C., "Controllability and Observability of Linear Dynamical Systems," Linear System Theory and Design, Oxford Univ. Press, New York, 1984, pp. 183-184.

[30] Anderson, B., and Moore, J., "The Standard Regulator Problem - 2," Optimal Control: Linear Quadratic Methods, edited by Kailath, T., Prentice-Hall, Englewood Cliffs, NJ, 1990, pp. 35-56.

[31] Khalil, H., "Advanced Stability Analysis," Nonlinear Systems, 3rd ed., Prentice-Hall, Upper Saddle River, NJ, 2002, pp. 312-322. 
This article has been cited by:

1. Robert A. E. Zidek, Alberto Bemporad, Ilya V. Kolmanovsky. Optimal and receding horizon drift counteraction control: Linear programming approaches 2636-2641. [Crossref]

2. Christopher D. Petersen, Frederick Leve, Ilya Kolmanovsky. 2016. Underactuated Spacecraft Switching Law for Two Reaction Wheels and Constant Angular Momentum. Journal of Guidance, Control, and Dynamics 39:9, 2086-2099. [Abstract] [Full Text] [PDF] [PDF Plus] 